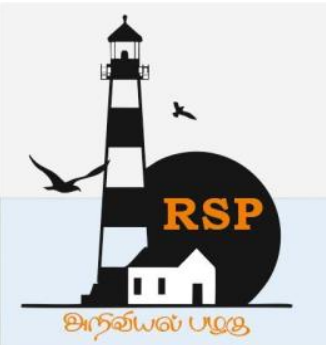

INTERNATIONAL RESEARCH JOURNAL ON ADVANCED SCIENCE HUB

RSP SCIENCE HUB

(The Hub of Research Ideas)

Available online at www.rspsciencehub.com

Special Issue of First International Conference on Information Technology, Computing \& Applications (ICITCA 2021)

\title{
EduAR- an AR based Learning Application
}

Kshitij Jethe ${ }^{1}$, Aniket Hedau ${ }^{2}$, Rohit Kolankar ${ }^{3}$, Sahil Dhoble ${ }^{4}$,Divyanshu Mataghare ${ }^{5}$

1,2,3,4,5 Department of Information Technology, S.B. Jain Institute of Technology, Management and Research, Nagpur, India.

kshitijj.it@sbjit.edu.in ${ }^{1}$,aniketh.it@sbjit.edu.in ${ }^{2}$, rohitk.it@sbjit.edu.in ${ }^{3}$

\begin{abstract}
Being one of the hot technologies of the era Augmented reality is exponentially developing various day to day activities and learning application is one of them. EduAR is an android application that overlays the virtual objects in a physical environment. EduAR as the title suggests it will be related to education and Augmented reality. Our project will be basically for preschool and kindergarten kids. In this application we are going to provide two main features, User will put camera on the particular word for alphabet and there will be a $3 D$ visual of the particular word in the form of image it will be a static image but will be presented are displayed in a magical way as simplifying and visualizing something new leads to easier understanding. Another feature is that the user will put a camera on the particular object or image and then it will display on screen what object is it children can have fun using this application to explore new things. This application will mainly focus on the development of young children to help them prepare for preschool and beyond.
\end{abstract}

Keywords: Augmented Reality, Learning, Education, real time, less efforts.

\section{Introduction}

It is the difficult challenge to visualize how any object looks. Augmented Reality (AR) is a technology where integration between digital elements with the real world in real time and follows the circumstances surrounding the environment. 'EduAR' is an android application that overlays the virtual object in a physical environment. EduAR as the title suggests it will be related to education and Augmented Reality this application will be basically for preschool and kindergarten kids. Augmented reality has an amazing ability to solve real life problems. Problems are mainly based on two factors i.e. time and money.[1-5].

\subsection{Main features of Application:-}

User will put a camera on the particular word for the alphabet and there will be a 3D visual of the particular word in the form of image. It will help for simplifying and visualizing this in an easier way. User will put a camera on the particular object or image and then it will be displayed on the screen, what object it is, children can have fun using this application to explore new things.

This application mainly focuses on the development of young children to help them prepare and beyond. A vehemence to engage with AR experiences is noted in many research studies while users report feeling higher satisfaction, having more fun, and being more willing to repeat the AR experiences.[6-10].

\section{A. Objectives}

- To provide virtual learning experience to a user.

- To provide long term memory retention.

- To provide a facility to have fun while learning and exploring. 
www.rspsciencehub.com

- To provide the user the visual of the size, shape and color of a particular object.

\section{Literature Survey}

Augmented Reality (AR) is integration of 3-D virtual objects into 3-D real environment in real time. It is the variation of Virtual Environments, or virtual reality. It describes the problems and issues that were encountered while developing Augmented Reality systems. It also describes the details characteristics of Augmented Reality including the detailed discussion of the compensation between optical and video blending approaches. The medical, visualization, path planning, entertainment and military applications have been explored. Summaries of the approaches taken so far to overcome problems and speculates on exploration of future. In Virtual Environment (VE) technologies the user gets completely immersed inside the virtual environment and the user is not able to see the real world around him, it allows the user to see the real world but virtual objects superimposed upon real world.[1].Computers in the past several decades are everywhere and have changed our work and lives. The transformation from traditional work with physical objects to the generation of computers has not been so easy. Now the revolution of computer is changing, rather than watching television monitor or typing on a keyboard, Augmented Reality let the people use familiar products in a different way. For example, Doctors can examine patients by viewing images, Children can make their own Lego constructions, and engineers can use ordinary papers drawings to communicate. This various approaches have a common goal that enable people to take advantage

\section{Proposed Work}

of their skills and benefitting the world, the main goal is to augment objects in physical world by enhancing them with digital information and communication capabilities. While designing augmented reality applications, the main considerations must be to integrate real and virtual, the application must choose the best combination of techniques for detecting all information from the real world.[2]. In today's world most of the systems use virtual reality technique hardware to see virtual objects. The Virtual Reality (VR) based applications simulate physical presence in the real world, as well as in imaginary worlds. Augmented Reality (AR) combines virtual and real environment, Augmented Reality (AR) is based on coupling between the virtual and the real object. The hardware needed for VR experience is still costly. Virtual environment does not have the same probable causes as training and working in the real world, which means that person might not do well in the real world as compared to a virtual environment. VR systems help students to walk around the surfaces and see how the variables interrelate. There are many fields in which AR is used such as education, military, commerce, business etc.[3-6]. Augmented Reality combines real and virtual environments, in past few years augmented reality has been used in many domains for multiple purposes; AR is used as an assistive system for performing various human tasks. Augmented reality (AR) has demonstrated to be useful in increasing the productivity and authenticity of the tasks especially in the domains related to surgery and aeroplane manufacturing.[4$8]$.

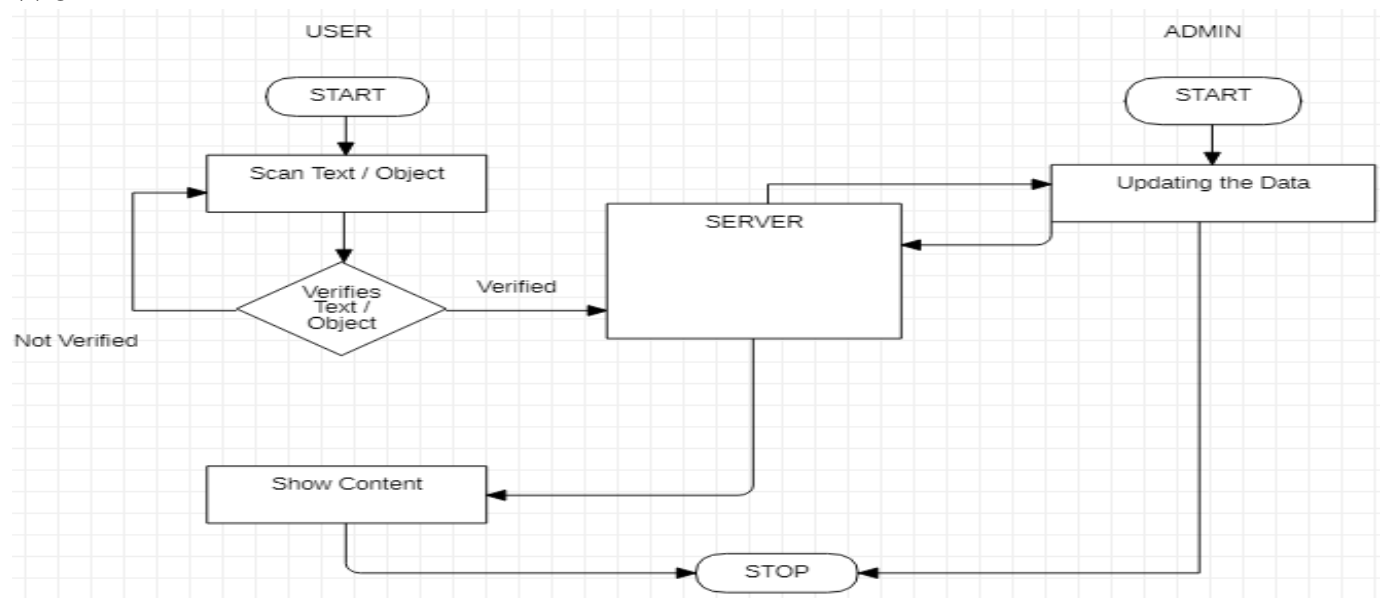

Fig.1.Flow of the System 
www.rspsciencehub.com

Our aim is to add digital graphical content to any word or alphabet. Students will get the concepts very easily as they would be able to explore the 3D models for particular words or alphabet and hence the visual communication will lead to proper understanding.
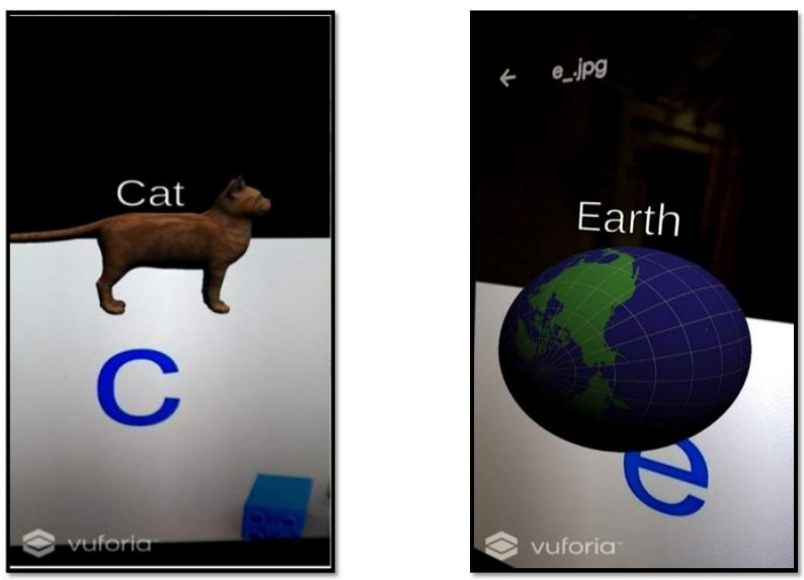

\section{Fig.2.Text Detection and Visualizing 3D Models}

Module 1: Text Detection and Visualization of 3D Models.

Module 2: Object Detection and Text Generation. The Fig.1. (Flowchart) shows the flow of our application. First, the user will open the application and will scan the letter or alphabet on a word application or in an image format. After that, the data will be searched on the server and if it's present the $3 \mathrm{~d}$ model will be shown on the screen of the user. The major work of the admin is to create new objects and update the database and make more and more objects to provide better options to users. The figure shows the flow of our application and working.

\section{Implementation}

Module 1: Text Detection and Visualizing 3D Models

Users have to put a camera on the particular alphabet and there will be a 3D visual of the particular word in the form of image. It will be a static image but will be presented or displayed in a 3D way. And the same goes with the words, the user will put the camera over the word and scan it and the 3D model will be displayed. [9-14].

$\checkmark$ A text is being detected.

$\checkmark$ A text is being recognized.

$\checkmark$ A3D model is being visualized/ displayed.
Volume 03 Issue 05S May 2021

Module 2: Object Detection and Text Generation Users have to put a camera on the particular object and an object is being detected. The 3D text will be visualized by recognizing the object and the short description about the object will be displayed on screen.

$\checkmark$ An object is being detected.

$\checkmark$ An object is being recognized.

$\checkmark$ A 3D text is being visualized/ displayed after recognizing the object.

$\checkmark$ An object is being detected.

$\checkmark$ An object is being recognized.

$\checkmark$ A 3D text is being visualized/ displayed after recognizing the object.
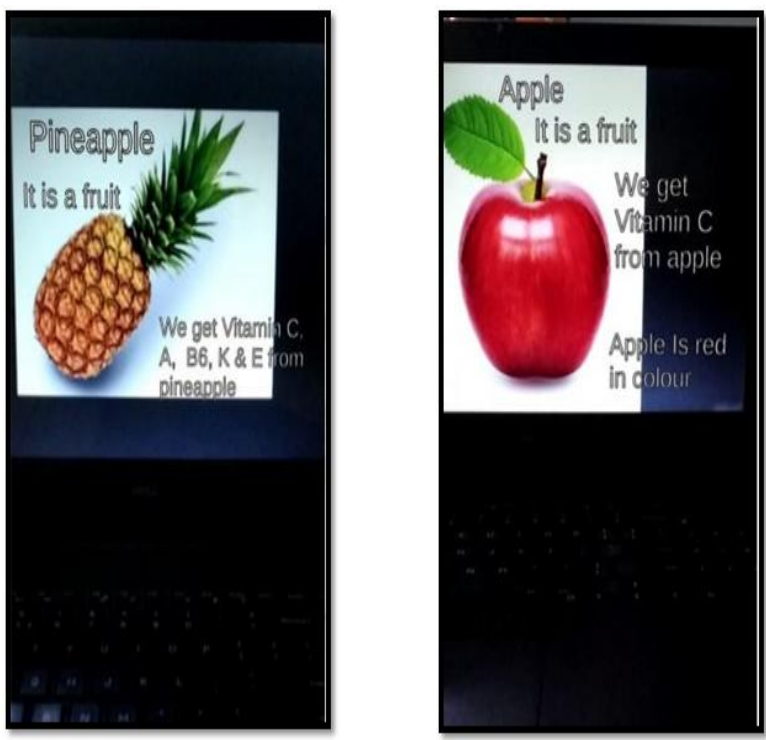

Fig.3. Object Detection and Text Generation Conclusion

We have designed a mobile application and completed its development by applying engineering knowledge which provides educational features by providing an Augmented Reality based learning platform. It would basically help the preschool and kindergarten kids to learn in a funny way, it detects the alphabet or word and displays the 3D visual of that particular object. Another feature implemented is that the user scans the object and application identifies the object and tells which object is it. We have used modern tools like Unity to implement the project. During the development of the project, we understood the importance of individual and teamwork by the project development and management. While presenting our project in various seminars we have enhanced our 
www.rspsciencehub.com

communication skills and displayed professional ethics which will result in lifelong learning.

\section{References}

\section{Papers:}

[1]. Ronald T. Azuma, "A Survey of Augmented Reality" Teleoperator and Virtual Environment (ISSN: 355-385) (August, 1997).

[2].Wendy E. Mackay, "Augmented Reality: Linking real and virtual worlds A new paradigm for interacting with computers:".

[3].Komal Gangurde, Bhagyashree Pardeshi, "Overlaying Virtual Object into Real World by Using Augmented Reality" International Research Journal of Engineering and Technology (IRJET) (Volume 3) ISSN: 23950056 (March, 2016).

[4].Shaunak S. Deshmukh, Chinmay M. Joshi, "3D Object Tracking and Manipulation in Augmented Reality:", International Research Journal of Engineering and Technology (IRJET) (Volume 5) ISSN: 2395-0056 (January, 2018).

[5]. Youngmin Park, Vincent Lepetit, "Multiple 3D Object Tracking for Augmented Reality" Foundation of UCN.

[6].R Silva, J. Oliveira "Introduction to Augmented Reality" National Lab for scientific Computation.

[7].Guidelines for Scanning Projects - Public Works \& services.

[8].Kevin Johnston, Jacques Ophoff "The Impact of an Augmented Reality Application on Learning Motivation of Students".

[9].Lavanya Sharma "Recent Advancement of Augmented Reality in Real Time Applications" Uttarakhand University.

[10].Robinett, Warren and Jannick Rolland."A Computational Model for the Stereoscopic Optics of a Head-Mounted Display." Teleoperators and Virtual Environments (Volume 1) (November 1992).

\section{Websites:}

[11].https://library.vuforia.com/tools/overview.ht $\mathrm{ml}$

[12].https://mw20.museweb.net/paper/developing -mobile-augmented-reality-with-unity-andvuforia/
Volume 03 Issue 05S May 2021

[13].https://programminghistorian.org/en/lessons/ creating-mobile-augmented-realityexperiences-in-unity

[14].https://library.vuforia.com/sites/default/files/ references/unity/annotated.html 